\title{
Research Paper: The Therapeutic Effect of Exercise on Anxiety and Bowel Oxidative Stress in the Maternal Separation Animal Model
}

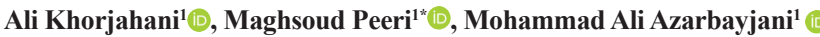

1. Department of Exercise Physiology, Central Tehran Branch, Islamic Azad University, Tehran, Iran.

Citation: Khorjahani, A., Peeri, M., \& Azarbayjani, M. A. (2020). The Therapeutic Effect of Exercise on Anxiety and Bowel Oxidative Stress in the Maternal Separation Animal Model. Basic and Clinical Neuroscience, 11(1), 69-78. http://dx.doi. org/10.32598/bcn.9.10.450

http://dx.doi.org/10.32598/bcn.9.10.450

\section{Article info:}

Received: 01 Aug 2018

First Revision: 25 Aug 2018

Accepted: 07 May 2019

Available Online: 01 Jan 2020

Keywords:

Maternal Separation (MS), Anxiety, Exercise, Brain, Gut, Oxidative stress

\begin{abstract}
A B S T RA C T
Introduction: According to evidence, Early-Life Stress (ELS), mood disorders, and medical comorbidities, i.e. Irritable Bowel Syndrome (IBS), are correlated; however, the direct contribution of ELS to IBS manifestations is less understood. The current study aimed at evaluating the effect of voluntary exercise on the mitochondrial dysfunction of the bowel fibroblasts, following the confirmation of anxiety behavior.

Methods: In this study, Postnatal Day (PND) rats underwent Maternal Separation (MS), as a valid animal model of the brain-gut axis dysfunction, in the days 2-14; three hours daily. On day 21, the study animals were divided into 4 groups, as follows: control, Running Wheel (RW) exercise, MS, and MS+RW groups. The study groups were housed in separate cages (4 rats per cage) until the onset of intervention. On day 60 , the elevated plus-maze was used to assess anxiety-like behaviors; the level of oxidative stress biomarkers, i.e. Reactive Oxygen Species (ROS), Glutathione (GSH), as well as Adenosine Triphosphate (ATP) was measured to determine the gut mitochondrial function.

Results: Findings revealed that ELS affected the gut energy metabolism in the studied rats; the negative effects of MS on anxiety and the gut mitochondrial dysfunction decreased via RW exercise during adolescence.

Conclusion: Overall, anxiety behaviors and ROS production, leading to increased GSH and ATP levels, improved after RW exercise; this significantly impacts the function of colon secretory mitochondria. According to the positive effects of RW exercise on mitochondrial dysfunction in an ELS animal model, a potential relationship was found between the brain and gut in the study rats.
\end{abstract}




\section{Highlights}

- Maternal separation is a valid animal model of brain-gut axis dysfunction in rats.

- Behavioral abnormalities were associated with mitochondrial dysfunction.

- The negative impact of maternal separation on the bowel mitochondrial function and animals' behavior reduced following the voluntary exercise.

\section{Plain Language Summary}

According to evidence, early-life stress, mood disorders, and some medical conditions, such as Irritable Bowel Syndrome (IBS), are correlated; IBS is among the commonest disabling diseases. This gastrointestinal illness is addressed by episodes of abdominal pain and bowel bloating. Unfortunately, the effect of antidepressant and anxiolytic agents, as well as other drug therapies on IBS treatment is unclear. Using a rat-model evaluation, current study findings indicated that the negative impacts of Maternal Separation (MS) on anxiety and the mitochondrial dysfunction of the gut could remarkably be controlled via Running Wheel (RW) exercise with no side effects.

\section{Introduction:}

arly-Life Stress (ELS) and stress-related psychiatric disorders are correlated with a disabling nature and a prevalent status (O’Mahony, Hyland, Dinan, \& Cryan, 2011). Prior research indicated that mood disorders are accompanied by other comorbid conditions in the periphery organs, like the heart and gut (Barrett et al., 2012; Clarke, Cryan, Dinan, \& Quigley, 2012; Eutamene et al., 2007; Klooker et al., 2009). Irritable Bowel Syndrome (IBS) is a common gastrointestinal illness addressed by the episodes of abdominal pain and bloating; in common sense, it is the brain-gut axis disorder (Zhao \& Qian, 2014). This condition's worldwide prevalence of $10 \%-15 \%$ is the most important reason for patients being visited by gastroenterologists. Furthermore, the prevalence of IBS is enhanced proportionately to Maternal Separation (MS) during childhood (Lowman, Drossman, Cramer, \& McKee, 1987).

According to evidence, the nature and severity of symptoms, physiological complications, and psychiatric disorders significantly affect IBS treatment. However, the effect of antidepressant and anxiolytic agents, as well as other pharmacotherapies on IBS treatment, remains unclear (Ford, Talley, Schoenfeld, Quigley, \& Moayyedi, 2008). Some studies have suggested physical exercise, as a novel and more efficacious method without adverse effects to concurrently treat IBS and anxiety (Carek et al., 2011; Herring et al., 2010).
Additionally, physical exercise increases the average life-span and improves health in humans (Radak, Chung, $\&$ Goto, 2008). Besides, other data have proven that exercise ameliorates the negative impact of MS stress on the behavior and incidence of oxidative stress in adult male rats (Radak, Taylor, Ohno, \& Goto, 2001). An animal model study on male rats indicated that Running Wheel (RW) exercise could descend depressive-like behaviors, and reduce the expression of immune genes in the hippocampus (Sadeghi, Peeri, \& Hosseini, 2016).

Evidence indicates that there are numerous mechanisms through which ELS induces its negative effects on the development of the brain and the formation of behavioral abnormalities (Herring et al., 2010). Studies indicated a correlation between oxidative stress and mitochondrial dysfunction and the pathophysiology of ELS-induced disorders (Taft \& Keefer, 2016). Moreover, based on preclinical data, psychopathologies, such as depressive- and anxiety-like behaviors, are irritated following the experimental induction of colitis, indicating the bidirectional relationship between the gut and brain (Bercik et al., 2010). However, limited research explored the effects of ELS, exercise, and their interaction on the bowel mitochondrial function and anxietylike behaviors. Therefore, the current study aimed at examining whether the rats with ELS induced by MS can develop anxiety-like behaviors. Besides, we investigated is the relationship between behavioral changes and the gut mitochondrial function. Finally, we assessed whether the ELS consequences on gut mitochondrial function are controlled by RW exercise. For this purpose, the gut fi- 
broblasts, as an important component of the bowel for the isolation of mitochondria, were selected.

\section{Methods}

Twenty pregnant albinoes Wistar rats (gestation day 1) were obtained from the Pasteur Institute of Iran (Tehran, Iran) and housed under the standard laboratory conditions $\left(22 \pm 2^{\circ} \mathrm{C}\right.$ temperature, $50 \% \pm 10 \%$ humidity, $12: 12$ $\mathrm{h}$ light-dark cycle, and standard rodents' food and water ad libitum). All the study experiments were performed according to the National Institute of Health (NIH) Guide for the Care and Use of Laboratory Animals (NIH publication \# 80-23), as well as the institutional guidelines for animal care and use (issued by the Department of Exercise Physiology, Central Tehran Branch, Islamic Azad University, Tehran, Iran).

Twenty pregnant rats were used in this study. Six male offspring were obtained for experiments and subsequently introduced to the MS paradigm based on the previously-published protocol (Amini-Khoei et al., 2017; Daniels, Marais, Stein, \& Russell, 2012). The birthday was considered as the Postnatal Day 0 (PND 0 ), and then, within the PNDs 2 to 14, the pups experienced maternal separation every day for 180 minutes, beginning at 09:00 AM. On PND 14, they were returned to their mothers. Four male rats were assigned to each group in a cage until testing on PNDs 60-62. All study rats were healthy and with no signs of sickness or death during the experiments.

The study rats were equally assigned to 4 groups of 10 -sample groups after the acclimation period. The groups were as follows: 1 . The control (had no access to the RW); 2. RW group; 3. MS group; and 4. MS+RW treatment group. After the completion of the RW intervention on PND 60, behavioral tests were performed on the study rats ( $\mathrm{n}=6-7$ per group). Furthermore, on PND 60 , the experimental group rats ( $n=3-4$ per group) were beheaded under mild anesthesia, and the bowel was removed on an ice surface for biochemical assay.

On PND 21, RW exercise protocol was performed on the randomly selected rats ( $\mathrm{n}=10$ per group). Accordingly, after a week of acclimation to the RW apparatus, MS rats underwent RW based on the Miladi-Gorji investigation protocol (Miladi-Gorji, Rashidy-Pour, \& Fathollahi, 2012). Each exercise cage that housed a pair of MS rats was separated with perforated Plexiglas from the neighbor cage to avoid animals' isolation and facilitate communication, and the RW apparatus was embedded in each cage. Similar conditions were provided for the control group, except for access to the RW. The Plexiglas wheels were $105 \mathrm{~cm}$ in circumference and $10 \mathrm{~cm}$ in width, with 5-g freely rotation (Novidan Tab, Iran). The rotation of the wheel was monitored hourly by a magnetic switch connected to and a counter placed outside the cage. There was no time limitation for using the RW apparatus, and the study rats were housed under the same conditions for 32 days until PND 60; their daily running distance was recorded in kilometers.

On RW exercise final day, behavioral tests were performed in rats (Pellow \& File, 1986). The Elevated Plus Maze (EPM) relies on the natural fear of mice of open, unprotected, and elevated areas (Suo et al., 2013). The black Plexiglas-made EMP had two open $(50 \times 10 \mathrm{~cm})$ and two closed $(50 \times 10 \times 40 \mathrm{~cm})$ cross-shared arms connected by a central platform $(10 \times 10 \mathrm{~cm})$. The apparatus was located $60 \mathrm{~cm}$ from the ground with dim light. First, the study rats were placed in the central zone facing the open arm and provided with a 5-minute chance to explore the maze as described previously (Sadeghi et al., 2016). A video camera was embedded above the EMP to record the sessions; the amount of time spent on the open arms plus the open arm entries were scored. To eliminate residual odors of the previous rat, the maze was cleaned between the tests with $70 \%$ ethanol.

On the final day of RW exercise treatment, behavioral tests were performed in the study rats according to the protocol of Amini-Khoei et al., 2017. The passive behavior of rats (immobility time) was monitored, while they were forced to swim individually in a $50 \times 20 \mathrm{~cm}$ (height $\times$ diameter) tall glass tank that $30 \mathrm{~cm}$ of it was water $\left(23 \pm 1^{\circ} \mathrm{C}\right)$ to conduct the Forced Swim Test (FST). Immobility is referred to when the animal floats in the water and makes only those movements necessary to keep its head above the surface.

We considered mitochondrial isolation from bowel fibroblasts. After the completion of behavioral interventions and treatment with exercise, the study animals were kept at fasting state for 24 hours, then were anesthetized. After opening the intestinal cavity, the bowel was immediately removed and washed with PhosphateBuffered Saline (PBS). The obtained tissue was quickly transferred to the DMEM medium, and after gently cutting, it was transferred to the Falcon Tube, and collagenase Intravenous (IV) (IV) was added to the medium (6-7 min). Then, the medium was filtered, and DMEM was re-added to the tube and placed instiller to isolate the bowel cells (10 min) uniformly. Then, the centrifuge was conducted at 400 RPM, and the supernatant was removed, and it was conducted again at $1000 \mathrm{~g}$; the 
resulting sediment was suspended again in the DMEM medium. The resulting sediment contained bowel fibroblasts. In the second stage, the extraction of mitochondrial fractions from fibroblasts was performed on the centrifuge at $2500 \mathrm{~g}$ for 10 minutes; then the supernatant was collected into a Falcon tube and centrifuged at 8000 $\mathrm{g}$ (10 $\mathrm{min})$, and the dark pallet tube containing the mitochondria was dispersed in terms of test in the desired buffer based on each test (Vicario et al., 2012; Wieckowski, Giorgi, Lebiedzinska, Duszynski, \& Pinton, 2009). To ensure the purity of the obtained mitochondrial suspension, MTT assay was conducted to confirm the results based on previous reports (Shaki et al., 2012).

Adenosine Triphosphate (ATP) assay was performed by the ATP assay kit using colorimetric assay according to the manufacturer's protocol (Abcam-83355). It is based on the phosphorylation of glycerol to generate a product that could be measured by Elisa-Reader at 570 nm based on a calibration standard curve.

The Dichloro-Dihydro-Fluorescein Diacetate (DCFHDA) assay was employed to measure the mitochondrial ROS production. Thus, $5 \mu \mathrm{L}$ of $10 \mu \mathrm{M}$ DCFH-DA was added to the supernatant, composed of respiratory buffer (Sonei et al., 2017). The amount of DCF, final fluorescent product, was determined at the excitation-emission wavelengths of 485 and $525 \mathrm{~nm}$, respectively (Amiri et al., 2016; Sonei et al., 2017).

Glutathione (GSH) level was measured by a spectrophotometer (UV-1601 PC, Shimadzu, Japan) at 412 nm using Thermo Scientific Pierce Ellman's Reagent (DTNB) following the emergence of yellow color based on calibration standard curve (Hosseini, Shaki, GhaziKhansari, \& Pourahmad, 2014; Shaki et al., 2012).

All obtained data were expressed as Mean \pm SD. Oneway Analysis of Variance (ANOVA), followed by Tuck-

Table 1. The Effect of RW exercises on ROS formation

\begin{tabular}{ccr}
\hline Groups & \multicolumn{2}{c}{ ROS Formation (\%) } \\
\cline { 2 - 3 } Control & $15 \mathrm{~min}$ min & $13 \pm 4$ \\
RW & $4 \pm 2$ & $8 \pm 3$ \\
Maternal Separation (MS) & $4 \pm 2$ & $50 \pm 9$ \\
MS+RW & $32 \pm 6$ & $19 \pm 5$
\end{tabular}

ey's posthoc test, was employed to make between-group comparisons. The level of significance was set at $\mathrm{P}<0.05$.

\section{Results}

Anxiety-like behavior was assessed in the studied rats using the EPM test. In this test, the reduced frequency of the open arm entry and increased time spent in the closed arms indicate higher levels of anxiety. Using statistical analysis, it was determined that MS stress significantly causes anxiety-like behaviors. In other words, the number of entries to the open arm of apparatus in MS groups $\left[\mathrm{F}_{(3,27)}=10.275, \mathrm{df}=3, \mathrm{P}<0.001\right.$, Figure 1] was lower than that of the control group. Moreover, the time spent in the open arm was decreased in the MS groups, compared to the controls $\left[\mathrm{F}_{(3,27)}=17.930, \mathrm{df}=3, \mathrm{P}<0.001\right.$, Figure 1]. Besides, the achieved data revealed that the MS+RW group exhibited a significant increase in the open arm entry and the time spent in the open arms, compared to the MS groups, suggesting a significant reduction in the anxiety-like behaviors (Figure 1).

Increased immobility time in the FST was considered behavioral despair, i.e. a symptom of human depression (Amiri et al., 2016). One-way ANOVA results suggested a significant difference in the FST scores $\left[\mathrm{F}_{(3,27)}=38.462\right.$, $\mathrm{P}<0.001]$. According to Figure $1 \mathrm{~B}$, the immobility time of MS rats was significantly higher than that of the controls $(* * * \mathrm{P}<0.001)$. Besides, the immobility time was shorter in the RW-treated MS rats, compared with the controls ( $\left.{ }^{\#} \mathrm{P}<0.001\right)$.

As per Table1, ROS level increased time-dependently in the MS group, compared with the controls after 15 $\left[\mathrm{F}_{(3,11)}=47.896, \mathrm{df}=3, \mathrm{P}<0.001\right]$ and $60\left[\mathrm{~F}_{(3,11)}=32.641\right.$, $\mathrm{df}=3, \mathrm{P}<0.001]$ minutes. This finding indicates the involvement of oxidative stress in isolated mitochondria obtained from bowel fibroblasts. The ROS production rate had no significant elevation in the $\mathrm{MS}+\mathrm{RW}$ rats after a 60-minute exposure, compared to the controls $(\mathrm{P}>0.05)$. 

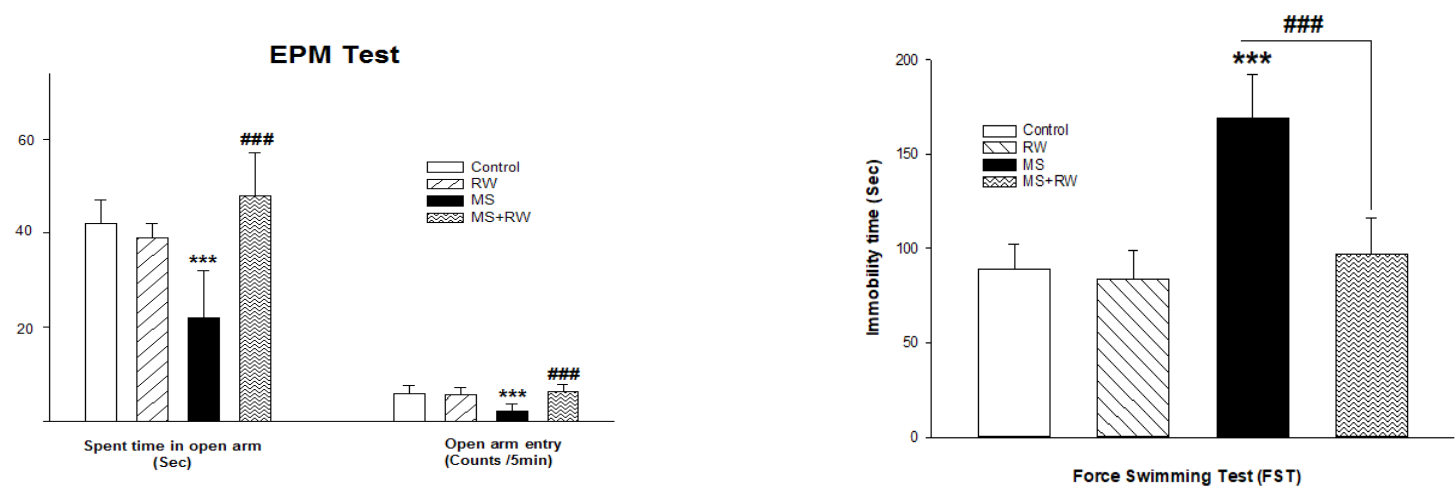

NEUROSCIENCE

Figure 1. A. The effect of RW exercise on anxiogenic-like behaviors B. The effect of RW exercise on depressive-like behaviors

The effect of chronic adolescent RW exercise on time spent on the open arm, open arm entry, and time spent in the center of EPM. Values are expressed as Mean $\pm \mathrm{SD}$ and were analyzed using one-way ANOVA followed by Tukey's posttest test ( $\mathrm{n}=7-8$ in all groups). ${ }^{* *} \mathrm{P}<0.001$ compared with the control group; ${ }^{\# \# \# P} \mathrm{P}<0.001$ compared with the MS groups.

The effect of chronic adolescent RW exercise on immobility time on OFT. Values are expressed as Mean \pm SD and were analyzed using one-way ANOVA followed by Tukey's posttest test ( $\mathrm{n}=7-8$ in all groups). ${ }^{* * *} \mathrm{P}<0.001$ compared with the control group; \#\# $\mathrm{P}<0.001$ compared with the MS groups.

A significant decrease was observed in the GSH level of the MS rats, in comparison with the controls $(\mathrm{P}<0.05)$. However, in the RW exercise groups, the GSH level had a significant elevation, compared to the MS group $\left[\mathrm{F}_{(3,11)}=22.941, \mathrm{df}=3, \mathrm{P}<0.001\right]$ (Figure 2).

The ATP level significantly decreased in the MS rats, compared to the controls $\left[\mathrm{F}_{(3,11)}=13.5, \mathrm{df}=3, \mathrm{P}<0.001\right]$. However, the RW exercise groups demonstrated a significant increase in ATP level, compared to the MS groups (Figure 3).

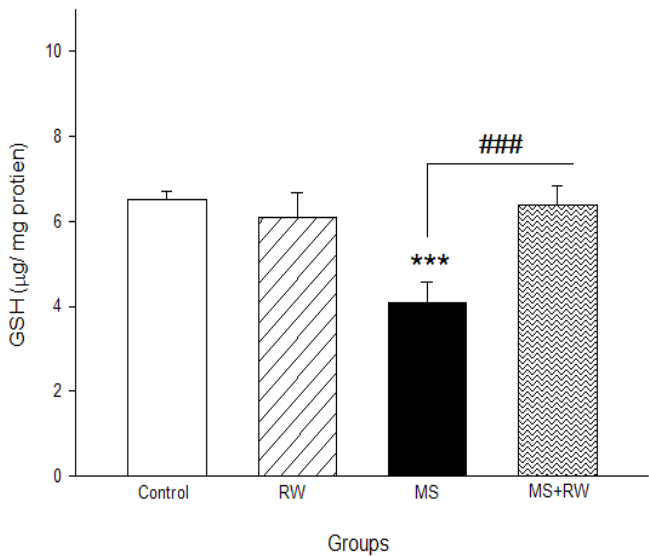

NEUR SCIENCE

Figure 2. The effect of RW exercises on GSH level

Values are expressed as Mean $\pm \mathrm{SD}$. ${ }^{* * *} \mathrm{P}<0.001$ compared with the control group and ${ }^{\# \#} \mathrm{P}<0.001$ compared with the MS group.
Reactive oxygen species were measured by fluorescent dye DCFH-DA. The change in fluorescence was determined at $485 \mathrm{~nm}$ for excitation and $525 \mathrm{~nm}$ for emission after 15 and 60 min incubation, using fluorometry.

\section{Discussion}

MS stress in rodents is a non-pharmacological method that could induce behavioral changes, such as depression, anxiety, and inducing visceral hypersensitivity

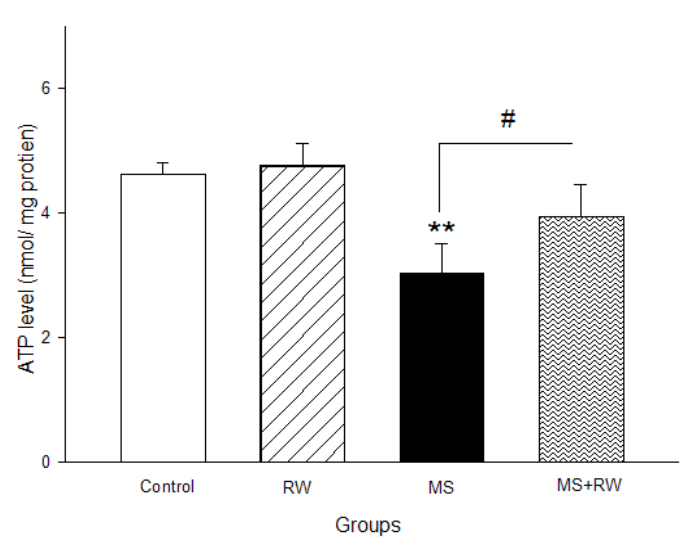

NEUR:SCIENCE

Figure 3. The effect of MS in childhood on mitochondrial ATP level

Values are expressed as mean $\pm \mathrm{SD}$. ${ }^{*} \mathrm{P}<0.05 ;{ }^{*} \mathrm{P}<0.01$; *** $\mathrm{P}<0.001$ compared with the control group and ${ }^{\#} \mathrm{P}<0.05$; \#\# $\mathrm{P}<0.01$; \#\# $\mathrm{P}<0.001$ compared with the MS group. 
to rectal distention (Carek et al., 2011; Sadeghi et al., 2016). The current study findings supported experiencing MS-induced anxiety- and depressive-like behaviors and mitochondrial dysfunction in bowel fibroblasts. The obtained data indicated that oxidative stress and antioxidant imbalance contribute to the negative effects of ELS on gut energy metabolism. Besides, RW exercise could reverse the ELS-induced anxiogenic behavior and abnormalities in the gut mitochondrial function.

Several lines of clinical and preclinical research have suggested that early life stress and trauma could alter GI motility, disrupt intestinal epithelial barrier, and activate gut mucosal inflammatory responses during adulthood (Cryan \& Holmes, 2005; Nicholl et al., 2008; O’Mahony et al., 2009; O’Malley, Julio-Pieper, Gibney, Dinan, \& Cryan, 2010). According to clinical data, 50\%-60\% of patients with IBS also have psychiatric disorders, like depression and anxiety (Vicario et al., 2012). According to the current study, the MS-treated group presented anxiety-like behaviors. Such behaviors were characterized by spending a shorter time on the open arms and less frequency of the open arms in the EPM. Besides, the achieved data suggested that RW exercise ameliorated anxiety-like behaviors in the study rats.

Vicario et al. argued that the reversible mitochondrial damage and upregulated corticotropin-releasing factor receptor type-1 are induced in the gut following the incidence of chronic social stress; they could cause IBSlike gut dysfunction (Vicario et al., 2012). Additionally, physical activity and exercise induce widespread neurobiological adaptations and could reduce anxiety-like behaviors, enhance neurogenesis via promoting IGF1 and BDNF activity (Droste et al., 2003; Schoenfeld, Rada, Pieruzzini, Hsueh, \& Gould, 2013), and reduce ACTH hormones and corticosterone levels (Hare, Beierle, Toufexis, Hammack, \& Falls, 2014; Wang, Chen, Lin, Jhong, \& Chen, 2014). A study reported the expression of the NR2A subunit of NMDA receptors increased following MS in the hippocampus, i.e. crucial in the incidence of depression- and anxiety-like behaviors. Moreover, the negative impacts of MS on behavior and mitochondrial function was controlled via RW in adult rats (Fattahi, Peeri, Azarbayjani, \& Hosseini, 2017). Therefore, MS could enhance glutamatergic signaling, which causes anxiety-like behaviors and mitochondrial dysfunction in bowel, similar to depressive-like behaviors.

Accordingly, evidence indicated that dysfunction in the mitochondrial bioenergetics influences the pathophysiology of psychiatric disorders; i.e. depression and anxiety via different routes, such as the activation of oxida- tive stress or inflammatory pathways, the activation of NMDA receptor, and altering the plasticity of neurons (Brown \& Bal-Price, 2003; Gardner \& Boles, 2011; Klinedinst \& Regenold, 2015; Morava \& Kozicz, 2013). A study revealed the induction of mitochondrial dysfunction and oxidative stress in the bowel fibroblasts of MS adult rats (Vicario et al., 2012). The results of ROS production demonstrated that the rate of ROS production was higher in MS rats, compared with the controls in the mitochondria obtained from bowel; therefore, ROS overproduction in mitochondria is induced following the early-life maternal separation, i.e. associated with mitochondria dysfunction and oxidative stress in the bowel cells (Table 1).

In line with these results, the findings of a study indicated that MS exposure caused a significant decrease in GSH level, which supports increased ROS production. Furthermore, another study suggested that MS negatively impacted GSH level, as the main antioxidant system, and adversely affects mitochondrial permeability transition pore opening. ATP levels in MS rats were decreased compared to the control groups. This finding indicates that early life can disrupt the metabolism of energy in the bowel mitochondria isolated from fibroblast in adult rats.

The present investigation revealed the significant reduction of ROS production on the isolated mitochondria obtained from bowel tissue following treatment with RW exercise (Bachur et al., 2007; Salim et al., 2010). Improving the antioxidant system following RW exercise and decreasing superoxide radical production or ROS production have a protective role against MS-induced changes. Besides, the study findings were consistent with those of other studies indicating exercise increased GSH level (Bachur et al., 2007; Hovanloo, Hedayati, Ebrahimi, \& Abednazari, 2011; Oh-Ishi et al., 1994; Rezin et al., 2009; Sonei et al., 2017). The inconsistency between the results is due to the difference in the antioxidant and oxidant scavenger pattern or difference in the intensity and duration of exercises. In terms of recommendations, finding indicated the induction of mitochondrial dysfunction in the bowel fibroblast by ELS-derived stress; thus, the impact of exercise on anxiety might be affected by the intensity and duration of exercise, resting time after exercise and the severity of the psychiatric disorder.

\section{Conclusion}

the current study results suggested that experiencing stress due to early-life maternal separation is a risk factor for medical comorbidities; i.e. bowel complications in adulthood; however, t RW exercise during adolescence 
indicated anxiolytic and protective effects on the negative outcomes of ELS on the mitochondrial function of the bowel.

\section{Ethical Considerations}

\section{Compliance with ethical guidelines}

All institutional and national guidelines for the care and use of laboratory animals were considered.

\section{Funding}

There was no funding for the study.

\section{Authors' contributions}

All authors contributed in preparing this paper.

Conflict of interest

The authors declared no conflicts of interest.

\section{References}

Amini-Khoei, H., Mohammadi-Asl, A., Amiri, S., Hosseini, M.J., Momeny, M., \& Hassanipour, M., et al. (2017). Oxytocin mitigated the depressive-like behaviors of maternal separation stress through modulating mitochondrial function and neuroinflammation. Progress in Neuro-Psychopharmacology and Biological Psychiatry, 76, 169-78. [DOI:10.1016/j.pnt pbp.2017.02.022] [PMID]

Amiri, S., Amini-Khoei, H., Mohammadi-Asl, A., Alijanpour, S. Haj-Mirzaian, A., \& Rahimi-Balaei, M., et al. (2016). Involvement of D1 and D2 dopamine receptors in the antidepressant-like effects of selegiline in maternal separation model of mouse. Physiology \& Behavior, 163, 107-14. [DOI:10.1016/j. physbeh.2016.04.052] [PMID]

Amiri, S., Haj-Mirzaiain, A., Momeny, M., Amini-Khoei, H., Rahimi-Balaei, M., \& Poursaman, S., et al. (2017). Streptozotocin induced oxidative stress, innate immune system responses and behavioral abnormalities in male mice. Neuroscience, 340, 373-83. [DOI:10.1016/j.neuroscience.2016.11.003] [PMID]

Bachur, J. A., Garcia, S. B., Vannucchi, H., Jordao, A. A., Chiarello, P. G., \& Zucoloto, S. (2007). Anti-oxidative systems in rat skeletal muscle after acute physical exercise. Applied Physiology, Nutrition, and Metabolism, 32(2), 190-6. [DOI:10.1139/ h06-078] [PMID]

Barrett, E., Fitzgerald, P., Dinan, T. G., Cryan, J. F., Ross, R. P., \& Quigley, E. M., et al. (2012). Bifidobacterium breve with a-linolenic acid and linoleic acid alters fatty acid metabolism in the maternal separation model of irritable bowel syndrome. PLoS One, 7(11), e48159. [DOI:10.1371/journal.pone.0048159] [PMID] [PMCID]
Bercik, P., Verdu, E. F., Foster, J. A., Macri, J., Potter, M., \& Huang, X., et al. (2010). Chronic gastrointestinal inflammation induces anxiety-like behavior and alters central nervous system biochemistry in mice. Gastroenterology, 139(6), 2102-2112. e2101. [DOI:10.1053/j.gastro.2010.06.063] [PMID]

Brown, G. C., \& Bal-Price, A. (2003). Inflammatory neurodegeneration mediated by nitric oxide, glutamate, and mitochondria. Molecular Neurobiology, 27(3), 325-55. [DOI:10.1385/ $\mathrm{MN}: 27: 3: 325$

Carek, P. J., Laibstain, S. E., \& Carek, S. M. (2011). Exercise for the treatment of depression and anxiety. The International Journal of Psychiatry in Medicine, 41(1), 15-28. [DOI:10.2190/PM.41.1.c] [PMID]

Clarke, G., Cryan, J., Dinan, T., \& Quigley, E. M. (2012). probiotics for the treatment of irritable bowel syndrome-focus on lactic acid bacteria. Alimentary Pharmacology \& Therapeutics, 35(4), 403-13. [DOI:10.1111/j.1365-2036.2011.04965.x] [PMID]

Cryan, J. F., \& Holmes, A. (2005). The ascent of mouse: advances in modelling human depression and anxiety. Nature reviews. Drug Discovery, 4(9), 775. [DOI:10.1038/nrd1825] [PMID]

Daniels, W. M., Marais, L., Stein, D. J., \& Russell, V. A. (2012). Exercise normalizes altered expression of proteins in the ventral hippocampus of rats subjected to maternal separation Experimental Physiology, 97(2), 239-47. [DOI:10.1113/expphysy iol.2011.061176] [PMID]

Droste, S. K., Gesing, A., Ulbricht, S., Muiller, M. B., Linthorst, A. C., \& Reul, J. M. (2003). Effects of long-term voluntary exercise on the mouse hypothalamic-pituitary-adrenocortical axis. Endocrinology, 144(7), 3012-23. [DOI:10.1210/en.20030097] [PMID]

Eutamene, H., Lamine, F., Chabo, C., Theodorou, V., Rochat F., \& Bergonzelli, G. E., et al. (2007). Synergy between Lactobacillus paracasei and its bacterial products to counteract stress-induced gut permeability and sensitivity increase in rats. The Journal of Nutrition, 137(8), 1901-7. [DOI:10.1093/ jn/137.8.1901] [PMID]

Fattahi, M. F., Peeri, M., Azarbayjani, M., \& Hosseini, M. (2017). Voluntary exercise during adolescence mitigated negative the effects of maternal separation stress on the depressivelike behaviors of adult male rats: Role of NMDA receptors. Neurochemical Research, 43(5):1067-74. [DOI: 10.1007/s11064018-2519-6]

Ford, A. C., Talley, N. J., Schoenfeld, P. S., Quigley, E. M., \& Moayyedi, P. (2008). Efficacy of antidepressants and psychological therapies in irritable bowel syndrome: systematic review and meta-analysis. Gut, 58(3):367-78 [DOI:10.14309/00000434-200809001-01219]

Gardner, A., \& Boles, R. G. (2011). Beyond the serotonin hypothesis: mitochondria, inflammation and neurodegeneration in major depression and affective spectrum disorders. Progress in Neuro-Psychopharmacology and Biological Psychiatry, 35(3), 730-43. [DOI:10.1016/j.pnpbp.2010.07.030] [PMID]

Hare, B. D., Beierle, J. A., Toufexis, D. J., Hammack, S. E., \& Falls, W. A. (2014). Exercise-associated changes in the corticosterone response to acute restraint stress: evidence for increased adrenal sensitivity and reduced corticosterone response duration. Neuropsychopharmacology, 39(5), 1262. [DOI:10.1038/ npp.2013.329] [PMID] [PMCID] 
Herring, M. P., O'connor, P. J., \& Dishman, R. K. (2010). The effect of exercise training on anxiety symptoms among patients: a systematic review. Archives of Internal Medicine, 170(4), 321-31. [DOI:10.1001/archinternmed.2009.530] [PMID]

Hosseini, M.J., Shaki, F., Ghazi-Khansari, M., \& Pourahmad, J. (2014). Toxicity of copper on isolated liver mitochondria: impairment at complexes I, II, and IV leads to increased ROS production. Cell Biochemistry and Biophysics, 70(1), 367-81. [DOI:10.1007/s12013-014-9922-7] [PMID]

Hovanloo, F., Hedayati, M., Ebrahimi, M., \& Abednazari, H. (2011). Effect of various time courses of endurance training on alterations of antioxidant enzymes activity in rat liver tissue. Research in Medicine, 35(1), 14-9.

Klinedinst, N. J., \& Regenold, W. T. (2015). A mitochondrial bioenergetic basis of depression. Journal of Bioenergetics and Biomembranes, 47(1-2), 155-71. [DOI:10.1007/s10863-014-9584-6] [PMID]

Klooker, T. K., Braak, B., Painter, R. C., De Rooij, S. R., Van Elburg, R. M., \& Van Den Wijngaard, R. M., et al. (2009). Exposure to severe wartime conditions in early life is associated with an increased risk of irritable bowel syndrome: a population-based cohort study. The American Journal of Gastroenterology, 104(9), 2250. [DOI:10.1038/ajg.2009.282] [PMID]

Lowman, B. C., Drossman, D. A., Cramer, E. M., \& McKee, D. C. (1987). Recollection of childhood events in adults with irritable bowel syndrome. Journal of Clinical Gastroenterology, 9(3), 324-30. [DOI:10.1097/00004836-198706000-00017] [PMID]

Mashayekhi, V., Eskandari, M. R., Kobarfard, F., Khajeamiri, A., \& Hosseini, M. J. (2014). Induction of Mitochondrial Permeability Transition (MPT) pore opening and ROS formation as a mechanism for methamphetamine-induced mitochondrial toxicity. Naunyn-Schmiedeberg's Archives of Pharmacology, 387(1), 47-58. [DOI:10.1007/s00210-013-0919-3] [PMID]

Miladi-Gorii, H., Rashidy-Pour, A., \& Fathollahi, Y. (2012). Anxiety profile in morphine-dependent and withdrawn rats: effect of voluntary exercise. Physiology \& Behavior, 105(2), 195-202. [DOI:10.1016/j.physbeh.2011.08.010] [PMID]

Morava, É., \& Kozicz, T. (2013). Mitochondria and the economy of stress (mal) adaptation. Neuroscience $\mathcal{E}$ Biobehavioral Reviews, 37(4), 668-80. [DOI:10.1016/j.neubiorev.2013.02.005] [PMID]

Nicholl, B., Halder, S., Macfarlane, G., Thompson, D., O'brien, S., \& Musleh, M., et al. (2008). Psychosocial risk markers for new onset irritable bowel syndrome-results of a large prospective population-based study. PAIN®, 137(1), 147-55. [DOI:10.1016/j.pain.2007.08.029] [PMID] [PMCID]

O'Mahony, S. M., Marchesi, J. R., Scully, P., Codling, C., Ceolho, A.M., \& Quigley, E. M., et al. (2009). Early life stress alters behavior, immunity, and microbiota in rats: implications for irritable bowel syndrome and psychiatric illnesses. Biological Psychiatry, 65(3), 263-67. [DOI:10.1016/j.biopsych.2008.06.026] [PMID]

O'Malley, D., Julio-Pieper, M., Gibney, S. M., Dinan, T. G., \& Cryan, J. F. (2010). Distinct alterations in colonic morphology and physiology in two rat models of enhanced stress-induced anxiety and depression-like behaviour. Stress, 13(2), 114-22. [DOI:10.3109/10253890903067418] [PMID]

O'Mahony, S. M., Hyland, N. P., Dinan, T. G., \& Cryan, J. F. (2011). Maternal separation as a model of brain-gut axis dys- function. Psychopharmacology, 214(1), 71-88. [DOI:10.1007/ s00213-010-2010-9] [PMID]

Oh-Ishi, S., Kizaki, T., Yamashita, H., Ohira, Y., Sato, Y., \& Nagata, N., et al. (1994). Effects of Endurance Training on Mn-SOD Activity, Content, and mRNA Expression in RatSkeletal Muscle. (Portland Press Limited). Clinical and Experimental Pharmacology and Physiology, 24(5):326-32. [DOI:10.1042/cs087s084]

Pellow, S., \& File, S. E. (1986). Anxiolytic and anxiogenic drug effects on exploratory activity in an elevated plus-maze: a novel test of anxiety in the rat. Pharmacology Biochemistry and Behavior, 24(3), 525-9. [DOI:10.1016/0091-3057(86)90552-6]

Radak, Z., Chung, H. Y., \& Goto, S. (2008). Systemic adaptation to oxidative challenge induced by regular exercise. Free Radical Biology and Medicine, 44(2), 153-9. [DOI:10.1016/j.freeradbib omed.2007.01.029] [PMID]

Radak, Z., Taylor, A. W., Ohno, H., \& Goto, S. (2001). Adaptation to exercise-induced oxidative stress: from muscle to brain. Exercise Immunology Review, 7, 90-107.

Rezin, G. T., Gonçalves, C. L., Daufenbach, J. F., Fraga, D. B., Santos, P. M., \& Ferreira, G. K., et al. (2009). Acute administration of ketamine reverses the inhibition of mitochondrial respiratory chain induced by chronic mild stress. Brain Research Bulletin, 79(6), 418-21. [DOI:10.1016/j.brainresbull.2009.03.010] [PMID]

Sadeghi, M., Peeri, M., \& Hosseini, M. J. (2016). Adolescent voluntary exercise attenuated hippocampal innate immunity responses and depressive-like behaviors following maternal separation stress in male rats. Physiology \& Behavior, 163, 177183. [DOI:10.1016/j.physbeh.2016.05.017] [PMID]

Salim, S., Sarraj, N., Taneja, M., Saha, K., Tejada-Simon, M. V., \& Chugh, G. (2010). Moderate treadmill exercise prevents oxidative stress-induced anxiety-like behavior in rats. Behavioural Brain Research, 208(2), 545-52. [DOI:10.1016/j.bbr.2009.12.039] [PMID]

Schoenfeld, T. J., Rada, P., Pieruzzini, P. R., Hsueh, B., \& Gould, E. (2013). Physical exercise prevents stress-induced activation of granule neurons and enhances local inhibitory mechanisms in the dentate gyrus. Journal of Neuroscience, 33(18), 7770-7. [DOI:10.1523/JNEUROSCI.5352-12.2013] [PMID] [PMCID]

Shaki, F., Hosseini, M.-J., Ghazi-Khansari, M., \& Pourahmad, J. (2012). Toxicity of depleted uranium on isolated rat kidney mitochondria. Biochimica et Biophysica Acta (BBA)-General Subjects, 1820(12), 1940-50. [DOI:10.1016/j.bbagen.2012.08.015] [PMID]

Sonei, N., Amiri, S., Jafarian, I., Anoush, M., Rahimi-Balaei, M., \& Bergen, H., et al. (2017). Mitochondrial dysfunction bridges negative affective disorders and cardiomyopathy in socially isolated rats: Pros and cons of fluoxetine. The World Journal of Biological Psychiatry, 18(1), 39-53. [DOI:10.3109/15622975.2016 .1149218] [PMID]

Suo, L., Zhao, L., Si, J., Liu, J., Zhu, W., \& Chai, B., et al. (2013) Predictable chronic mild stress in adolescence increases resilience in adulthood. Neuropsychopharmacology, 38(8), 1387. [DOI:10.1038/npp.2013.67] [PMID] [PMCID]

Taft, T. H., \& Keefer, L. (2016). A systematic review of diseaserelated stigmatization in patients living with inflammatory bowel disease. Clinical and Experimental Gastroenterology, 9, 49. [DOI:10.2147/CEG.S83533] [PMID] [PMCID] 
Vicario, M., Alonso, C., Guilarte, M., Serra, J., Martínez, C., \& González-Castro, A. M., et al. (2012). Chronic psychosocial stress induces reversible mitochondrial damage and corticotropin-releasing factor receptor type-1 upregulation in the rat intestine and IBS-like gut dysfunction. Psychoneuroendocrinology, 37(1), 65-77. [DOI:10.1016/j.psyneuen.2011.05.005] [PMID]

Wang, D.-C., Chen, T.-J., Lin, M.-L., Jhong, Y.-C., \& Chen, S.-C. (2014). Exercise prevents the increased anxiety-like behavior in lactational di-(2-ethylhexyl) phthalate-exposed female rats in late adolescence by improving the regulation of hypothalamus-pituitary-adrenal axis. Hormones and Behavior, 66 (4), 67484. [DOI:10.1016/j.yhbeh.2014.09.010] [PMID]

Wieckowski, M. R., Giorgi, C., Lebiedzinska, M., Duszynski, J., \& Pinton, P. (2009). Isolation of mitochondria-associated membranes and mitochondria from animal tissues and cells. Nature Protocols, 4(11), 1582. [DOI:10.1038/nprot.2009.151] [PMID]

Zhao, Y., \& Qian, L. (2014). Homocysteine-mediated intestinal epithelial barrier dysfunction in the rat model of irritable bowel syndrome caused by maternal separation. Acta Biochim Biophys Sin, 46(10), 917-9. [DOI:10.1093/abbs/gmu076] [PMID] 
This Page Intentionally Left Blank 